\title{
Generalized de Sitter space
}

\author{
Pedro F. González-Díaz \\ Centro de Física “Miguel Catalán,"” Instituto de Matemáticas y Física Fundamental, Consejo Superior de Investigaciones Científicas, \\ Serrano 121, 28006 Madrid, Spain \\ (Received 19 July 1999; published 23 December 1999)
}

\begin{abstract}
This paper deals with some two-parameter solutions to the spherically symmetric, vacuum Einstein equations which, we argue, are more general than the de Sitter solution. The global structure of one such spacetime and its extension to the multiply connected case have also been investigated. By using a six-dimensional Minkowskian embedding as its maximal extension, we check that the thermal properties of the considered solution in such an embedding space are the same as those derived by the usual Euclidean method. The stability of the generalized de Sitter space containing a black hole has been investigated as well by introducing perturbations of the Ginsparg-Perry type in the first order approximation. It has been obtained that such a space perdures against the effects of these perturbations.
\end{abstract}

PACS number(s): 04.20.Jb, 04.62.+v, 04.70.Dy

\section{INTRODUCTION}

In some respects de Sitter space is to cosmology what the hydrogen atom is to atomic physics. In most practical situations they both become the first example where any respective new formalism, description, or theory is brought to be checked out. Actually, since the very moment it was discovered one finds it difficult to encounter a paper on cosmology in which no mention is made of a de Sitter universe. Soon after its publication [1], Einstein sent a series of letters to de Sitter [2] in which he did not express entire approval of the de Sitter cosmology. Two main criticisms were raised by Einstein. First, since it could only apply without any "world material,' that is stars, he found the de Sitter metric to have no physical meaning, and secondly, Einstein argued that whereas one can always put a rigid circular hoop into a de Sitter universe at any sufficiently early, though nonzero time, there is no place in it at a time $t=0$. While the first criticism leaves the de Sitter universe only as a good approximation for a sufficiently tiny matter density (which actually is the real case), the second one is nothing but an expression of Einstein's disapproval of the existence of the big bang, an attitude which evidently overcomes the de Sitter scenario.

It is rather ironic, however, that in spite of Einstein's reservations, de Sitter obtained his cosmological solution by just introducing the cosmological term first considered by Einstein himself in the general-relativity field equations for a spherically symmetric spacetime without any matter, that is, by considering the simplest possible scanario meeting the essentials of Einstein's ideas. The key differential point between de Sitter and Einstein [3] universes is that in the latter a given matter density was explicitly included, setting a preferred value of the cosmological constant so that the resulting solution represented a static universe. On the contrary, in de Sitter space the value of the cosmological constant is completely arbitrary. In the absence of any matter, the Einstein equations can be written as

$$
R^{\mu \nu}=\Lambda g^{\mu \nu}
$$

with $\Lambda$ the cosmological constant. Assuming then a static general spherically symmetric metric

$$
d s^{2}=-e^{A} d t^{2}+e^{B} d r^{2}+r^{2} d \Omega_{2}^{2},
$$

with $d \Omega_{2}^{2}=d \theta^{2}+\sin ^{2} \theta d \phi^{2}$ the metric on the unit three sphere, we obtain $A=-B$ and hence the Schwarschild-de Sitter solution [4]

$$
e^{A}=e^{-B}=1-\frac{\Lambda r^{2}}{3}-\frac{2 m}{r},
$$

where the mass $m$ comes about as an integration constant. de Sitter's static solution arises when we set $m=0$ in Eq. (1.3), i.e., in the pure vacuum case.

From a slightly more technical point of view, the de Sitter space can be indentified [5] as a maximally symmetric space of constant positive curvature (positive Ricci scalar) which is a solution to the Einstein equations (1.1) with a positive cosmological constant $\Lambda>0$. It can be visualized as a five hyperboloid [6]

$$
-\mathrm{v}^{2}+w^{2}+x^{2}+y^{2}+z^{2}=\frac{3}{\Lambda},
$$

embedded in $E^{5}$, with the most general metric of the de Sitter space being then that which is induced in this embedding, i.e.,

$$
d s^{2}=-d \mathrm{v}^{2}+d w^{2}+d x^{2}+d y^{2}+d z^{2} .
$$

This has topology $R \times S^{4}$, invariance group $S O(4,1)$, and shows ten Killings vectors (four boosts and six rotations).

What we have hitherto described corresponds to a de Sitter space with simply connected topology. Recently, Gott and $\mathrm{Li}$ [7] have also considered the important case of a multiply connected de Sitter space. These authors imposed the identification symmetry of the universal covering of Misner space [5] to the five-hyperboloid visualizing the de Sitter space, so inducing a boost transformation in the region $w$ $>|\mathrm{v}|$ covered by the static metric of this space that makes this region multiply connected. In this way, the event horizon 
of the de Sitter space becomes a (Cauchy) chronology horizon which defines the onset of an interior nonchronal region which supports closed timelike curves (CTC's). The multiply connected de Sitter space can be used to implement new boundary conditions for the universe according to which, rather than being created from nothing, the universe created itself [7].

In this paper we argue that the static de Sitter space for all topologies actually corresponds to rather restricted solutions of the Einstein field equations for a spherically symmetric empty space which becomes curved only by the action of quantum vacuum effects. We show that there exist more general solutions to this problem, defined by two cosmological parameters, namely, the usual cosmological constant and a new constant vacuum term could be associated to vacuum effects whose backreaction over the spacetime geometry led to higher-derivative terms in the gravitational action.

The rest of the paper can be outlined as follows. In Sec. II we discuss the physical arguments that lead to obtaining a two-parameter generalized de Sitter solution, deriving the precise form of such a new solution. We then investigate the nature of the event horizons and the global structure of the new solution, and maximally extend the metric beyond the apparent horizons, both in the simply connected and multiply connected cases. The thermal properties associated with the existence of these event horizons are studied in Sec. III by using the usual Euclidean procedure and a new method based on replacing the Kruskal maximal extension by the sixdimensional embedding Minkowskian space as the background spacetime where we allow the propagation of a massless scalar field. In Sec. IV we investigate the effects that different types of Ginsparg-Perry perturbations [8] have in the generalized Schwarzschild-de Sitter space, concluding that this space is stable to the action of such perturbations in first order. Finally, we briefly summarize and conclude in Sec. V.

\section{A TWO-PARAMETER VACUUM SOLUTION}

We start this section by noticing the sense in which the de Sitter space cannot be considered as the most general solution to spherically symmetric Einstein equations for the pure vacuum case. As it was pointed out in the Introduction, de Sitter space can be visualized by embedding it in the five hyperboloid (1.4), with embedding Minkowski metric (1.5). However, the general embedding class for metrics of type (1.2), with $A=-B=\ln [1+\rho(r)]$ and $\rho(r)$ a given function of the radial coordinate $r$, has been shown by Stephani [9] to be 2 , and therefore one should consider a six-dimensional Minkowski space as the most general embedding for it. This would mean that one of the six degrees of freedom of the embedding space should become frozen in the case of the usual de Sitter metric, so pointing toward some lack of generality for this metric. In fact, let us take the function $\rho(r)$ to be given by $\rho(r)=-H^{2} r^{2}$, with $H=\sqrt{\Lambda / 3}$, and write the six-dimensional Minkowski metric as

$$
d s^{2}=-d z_{0}^{2}+d z_{i}^{2}, \quad i=1, \ldots, 5
$$

embedding the de Sitter manifold according to, e.g., the following coordinate transformations:

$$
\begin{gathered}
z_{0}=k \sqrt{1-H^{2} r^{2}} \sinh (t / k), \quad z_{1}=k \sqrt{1-H^{2} r^{2}} \cosh (t / k), \\
z_{2}=f(r), \\
z_{3}=r \cos \phi \sin \theta, \quad z_{4}=r \sin \phi \sin \theta, \quad z_{5}=r \cos \theta,
\end{gathered}
$$

where $k$ is an arbitrary, dimensional parameter to be fixed later, and the function $f(r)$, defining the new coordinate $z_{2}$, is given by [10]

$$
\left(\frac{d f}{d r}\right)^{2}=\left(f^{\prime}\right)^{2}=-\left[\frac{k^{2}(d \rho / d r)^{2}+4 \rho}{4(1+\rho)}\right] .
$$

Taking for the arbitrary parameter $k$ the inverse of the surface gravity of de Sitter space, $k=\kappa_{c}^{-1}=H^{-1}$, we get then from Eq. (2.5) that the function $f(r)$ becomes a constant, $f_{0}$, for de Sitter space, and hence it follows that the coordinate $z_{2}$ no longer is a degree of freedom for this space. Therefore, though the de Sitter static metric can still be visualized as a six hyperboloid, this is reducible to a five-hyperboloid defined for a rescaled cosmological constant $\Lambda_{R}=1 /\left(\Lambda^{-1}\right.$ $\left.-f_{0}^{2} / 3\right)$, and the embedding Minkowski metric becomes that of a five-dimensional space. In what follows we interpret this result as being an indication that the known de Sitter solution is not the most general solution that corresponds to general relativity with no matter for consistent nonzero quantummechanical vacuum fluctuations, for the following reason. Einstein equations were initially thought of as satisfying the requirement that they should permit flat spacetime as a particular solution in the absence of matter. By introducing the cosmological constant $\Lambda$, Einstein dropped [3] this requirement out and got a slightly more general set of equations which do not allow the existence of globally flat spacetime in the absence of matter, for then these equations become identical to Eq. (1.1).

Motivated by the facts that (i) any cosmological constant terms should all be associated to quantum fluctuations of vacuum [11], (ii) the gravitational Lagrangian must contain curvature squared corrections whenever it is considered quantum mechanically [12], and (iii) the physical dimensions of the cosmological constant terms should be that of the nonconstant part of the geometric terms containing the curvature in the gravitational action, we now go a step further by introducing in spherically symmetric spaces another vacuum term which we assume to depend on the radial coordinate $r$ as $\widetilde{Q} r^{2}$, where the constant $\widetilde{Q}$ can, in principle, be positive, negative, or zero. Similar to the curvature squared terms $R^{2}, R_{\mu \nu} R^{\mu \nu}$, etc., in higher-derivative gravity theories, the new constant $\widetilde{Q}$ ought to have the physical dimensions of a space curvature squared, namely, (length) ${ }^{-4}$. Disregarding the curvature squared terms in the left-hand side of the so modified field equations, we obtain 


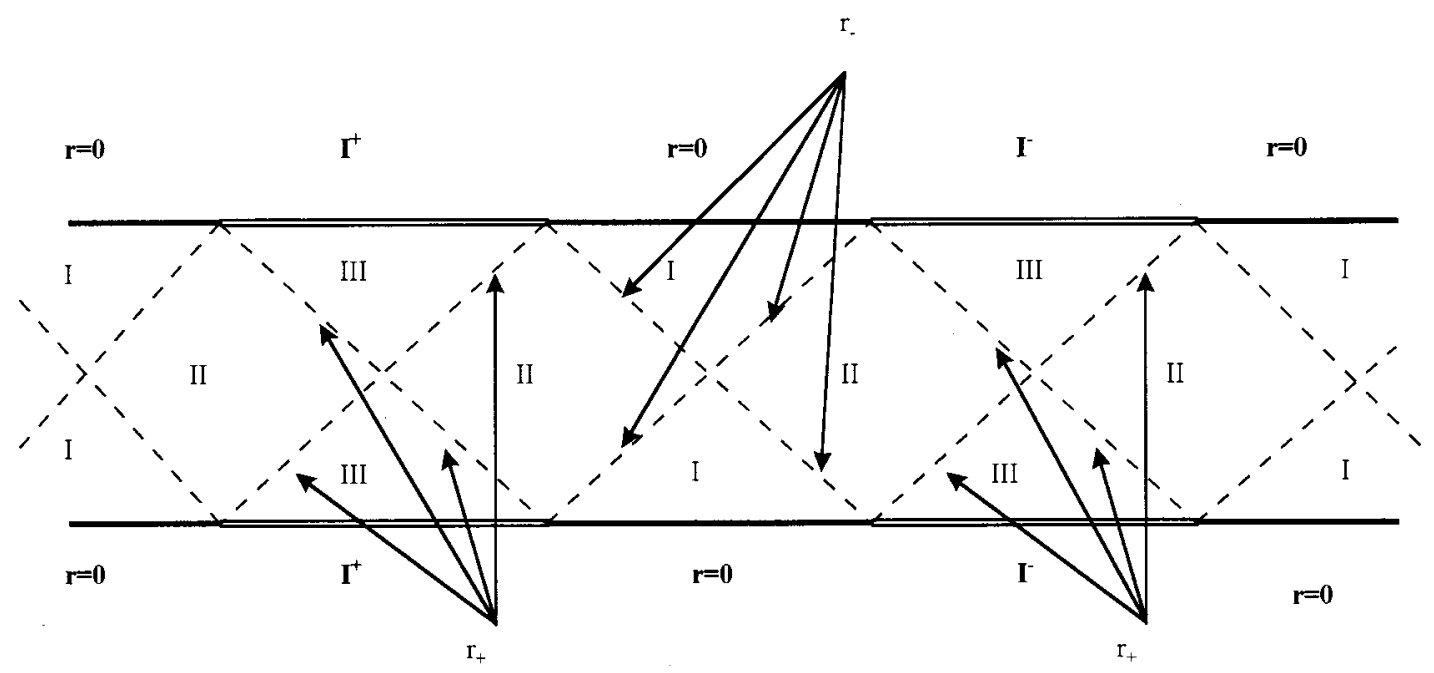

FIG. 1. The global structure of the generalized de Sitter space with negative $\widetilde{Q}$.

$$
R^{\mu \nu}=g^{\mu \nu}\left(\Lambda+\widetilde{Q} r^{2}\right) .
$$

Strictly speaking, one had to have considered field equations associated with higher-derivative theories with $R^{2}$ terms. However, in order to simplify our formulation, we restrict here ourselves to work in a semiclassical approximation where all quantum-mechanical effects are only taken into account in the right-hand side of the field equations.

Solutions obtained from Eq. (2.6) will then depend on two parameters, the cosmological constant $\Lambda$ and the new parameter $\widetilde{Q}$. The physical meaning of the cosmological constant is explicitly obtained in the quantum field theory context as being the (homogeneous and isotropic) ground state expectation value of some vacuum stress tensor. As to the physical interpretation of $\widetilde{Q}$, one may claim it to have an analogous origin as for $\Lambda$. Thus, $\widetilde{Q}$ ought to be quantum mechanically associated with some additional component of the vacuum stress tensor which would allow its value to be nonvanishing. On the other hand, given the explicit radial dependence assumed for the additional term which is displayed in Eq. (2.6), and the existence of a timelike singularity as one approaches $r=+\infty$ (see Fig. 1), one could expect that a nonvanishing $\widetilde{Q}$ would break homogeneity of the spatial sections at very large scales. This seems to raise the interesting possibility that corrections coming from $\widetilde{Q}$ might be considered as fluctuations around a homogeneous space, which eventually could be regarded as the seeds required for the formation of galaxies. Although in order to fix the precise form and physical meaning of the relation between the solutions containing the parameter $\widetilde{Q}$ and quantum vacuum fluctuations would require further detailed studies, it is still at this stage possible to conjecture that the generalized de Sitter spaces must describe additional quantum fluctuations which can be associated with virtual charged particles located at $r=0$. Note furthermore that the way in which $\widetilde{Q}$ will enter the relevant solutions [see, e.g., Eq. (2.7)] remains one of the electric charge in the Reissner-Nordström black hole solution.
Assuming then staticness, for the general spherically symmetric metric (1.2), we shall again obtain from the field equations (2.6) that $A=-B$, and from the equation for the spherical angle $\theta$,

$$
e^{A}=e^{-B}=1-\frac{\Lambda r^{2}}{3}-\frac{\widetilde{Q} r^{4}}{5}-\frac{2 m}{r},
$$

with $m$ an integration constant playing the role of an existing mass. Solution (2.7) describes a generalized Schwarzschild-de Sitter spacetime.

In what follows of this section and in the next one we consider the case $m=0$. Then, depending on the sign of the involved constants, we have distinct kinds of solutions. Setting $H=\sqrt{|\Lambda| / 3}$ and $Q^{4}=|\widetilde{Q}| / 5$, if $H^{2}>0$ and $\widetilde{Q}>0$, the resulting solution shows an event horizon at

$$
r_{H}^{2}=\frac{\sqrt{H^{4}+4 Q^{4}}-H^{2}}{2 Q^{4}}
$$

if $H^{2}>0$ and $\widetilde{Q}<0$, then there will be two horizons at

$$
r_{ \pm}^{2}=\frac{H^{2} \pm \sqrt{H^{4}-4 Q^{4}}}{2 Q^{4}}
$$

with $H^{2} \geqslant 2 Q^{2}$. Finally when $H^{2}<0$ and $\widetilde{Q}>0$ (generalized anti-de Sitter solution), we obtain also an event horizon at

$$
r_{H}^{2}=\frac{H^{2}+\sqrt{H^{4}+4 Q^{4}}}{2 Q^{4}} .
$$

In this paper, we will only consider the second of these particular solutions, i.e.,

$$
\begin{aligned}
d s^{2}= & -\left(1-H^{2} r^{2}+Q^{4} r^{4}\right) d t^{2} \\
& +\left(1-H^{2} r^{2}+Q^{4} r^{4}\right)^{-1} d r^{2}+r^{2} d \Omega_{2}^{2} .
\end{aligned}
$$


This metric can be embedded in the six-dimensional Minkowski space with metric (2.1) using the coordinate transformations

$$
\begin{gathered}
z_{0}=k \sqrt{1-H^{2} r^{2}+Q^{4} r^{4}} \sinh (t / k), \\
z_{1}=k \sqrt{1-H^{2} r^{2}+Q^{4} r^{4}} \cosh (t / k), \\
z_{2}=f(r), \\
z_{3}=r \cos \phi \sin \theta, \quad z_{4}=r \sin \phi \sin \theta, \quad z_{5}=r \cos \theta,
\end{gathered}
$$

where the function $f(r)$ again satisfies condition (2.5) which is singular at $r_{ \pm}$. Taking for the constant $k$ the inverse of the surface gravity which now becomes

$$
\kappa_{c}=\frac{r_{+}-r_{-}}{r_{+}^{2}}=\frac{2 Q^{2} \sqrt{H^{2}-2 Q^{2}}}{H^{2}+\sqrt{H^{4}-4 Q^{4}}},
$$

we get by using the equalities $Q^{2}=1 / r_{+} r_{-}, H^{2}=\left(r_{+}^{2}\right.$ $\left.+r_{-}^{2}\right) / r_{+}^{2} r_{-}^{2}$, and $\sqrt{H^{4}-4 Q^{4}}=\left(r_{+}^{2}-r_{-}^{2}\right) / r_{+}^{2} r_{-}^{2}$,

$$
\begin{aligned}
\left(f^{\prime}\right)^{2}= & -\left[\frac{r_{+} r_{-}^{3}\left(r_{+}^{2}+r_{-}^{2}\right)\left(r_{+}^{3} / r_{-}^{3}-r_{-} / r_{+}+2\right) r^{2}}{r_{+}^{2} r_{-}^{4}\left(r_{+}-r_{-}\right)^{2} g_{t t}}\right. \\
& \left.+\frac{r_{-}^{2}\left[\left(r_{+}-r_{-}\right)^{2}-2 r_{+}^{2}\left(r_{+}^{2} / r_{-}^{2}+1\right)\right] r^{4}+4 r_{+}^{2} r^{6}}{r_{+}^{2} r_{-}^{4}\left(r_{+}-r_{-}\right)^{2} g_{t t}}\right],
\end{aligned}
$$

which only vanishes when $Q$ approaches zero. It appears that all of the degrees of freedom of the six-hyperboloid are now being used and that, therefore, metric (2.9) is more general than the usual de Sitter metric in order to describe a spherically symmetric empty space which is subjected to quantum vacuum fluctuations.

The function $\left(f^{\prime}\right)^{2}$ is positive and nonsingular for $r$ $\leqslant r_{-}$, i.e., in the region of the generalized de Sitter space which can be thought of as a four-dimensional membrane immersed in a six-dimensional flat space, with the parametric equations given by

$$
\begin{gathered}
z_{1}^{2}-z_{0}^{2}=\frac{Q^{4} r_{+}^{4}}{H^{2}-2 Q^{2}}\left(1-H^{2} r^{2}+Q^{4} r^{4}\right), \\
z_{2}=f(r), \\
z_{3}^{2}+z_{4}^{2}+z_{5}^{2}=r^{2} .
\end{gathered}
$$

The chosen solution $H^{2}>0, \widetilde{Q}<0$ is valid only for $H^{2}$ $\geqslant 2 Q^{2}$. As $H^{2}$ approaches $2 Q^{2}$ one obtains an extreme generalized de Sitter space, a case which we shall briefly discuss later on.

The transformations (2.10) are not unique and cover only the region $z_{1}>\left|z_{0}\right|$ of the Minkowski space. There could actually be four distinct transformations that together cover completely the Minkowski space, namely,

$$
\begin{aligned}
& z_{0}= \pm k \sqrt{1-H^{2} r^{2}+Q^{4} r^{4}} \sinh (t / k) \\
& z_{1}= \pm k \sqrt{1-H^{2} r^{2}+Q^{4} r^{4}} \cosh (t / k)
\end{aligned}
$$

plus another two with the coordinates $z_{1}$ and $z_{0}$ interchanged $\left(z_{1} \leftrightarrow z_{0}\right)$. This is exactly the same situation we are going to find when extending from metric (2.9) to its associated Kruskal metric [10]. In fact, metric (2.9) is geodesically incomplete because of the presence of the event horizons at $r_{ \pm}$, as given by Eq. (2.8). This geodesic incompleteness can be avoided by using the Kruskal technique [5,13], and therefore we first introduce the "tortoise" coordinate

$$
\begin{aligned}
r^{*}= & \int \frac{d r}{1-H^{2} r^{2}+Q^{4} r^{4}}=\frac{r_{+}^{2} r_{-}^{2}}{2\left(r_{+}^{2}-r_{-}^{2}\right)}\left[\frac{1}{r_{+}} \ln \left(\frac{r_{+}-r}{r_{+}+r}\right)\right. \\
& \left.-\frac{1}{r_{-}} \ln \left(\frac{r_{-}-r}{r_{-}+r}\right)\right]
\end{aligned}
$$

and then the advanced and retarded coordinates

$$
V=t+r^{*}, \quad W=t-r^{*},
$$

so that the metric becomes

$$
d s^{2}=-\left(1-H^{2} r^{2}+Q^{4} r^{4}\right) d V d W+r^{2} d \Omega_{2}^{2},
$$

with the radial coordinate $r$ defined by

$$
r^{*}=\frac{1}{2}(V-W)
$$

In the case that $Q^{2}<H^{2} / 2$, we introduce now the new coordinates $V^{\prime}$ and $W^{\prime}$ by means of the definitions

$$
V^{\prime}=\tan ^{-1}\left[\exp \left(\kappa_{c} V\right)\right], \quad W^{\prime}=\tan ^{-1}\left[-\exp \left(\kappa_{c} W\right)\right],
$$

and hence we obtain the Kruskal metric for a generalized de Sitter space with negative constant $\widetilde{Q}$, that is,

$$
d s^{2}=-\left(1-H^{2} r^{2}+Q^{4} r^{4}\right) \frac{4 d V^{\prime} d W^{\prime}}{\kappa_{c}^{2} \sin \left(2 V^{\prime}\right) \sin \left(2 W^{\prime}\right)}+r^{2} d \Omega_{2}^{2},
$$

where $\kappa_{c}$ is the surface gravity defined in Eq. (2.13), and $r$ is defined implicitly by the relation

$$
\tan V^{\prime} \tan W^{\prime}=-\left[\left(\frac{r_{+}-r}{r_{+}+r}\right)^{r_{-} / r_{+}}\left(\frac{r_{-}+r}{r_{-}-r}\right)\right]^{r_{-} /\left(r_{+}+r_{-}\right)} .
$$

The maximal extension is therefore obtained taking metric (2.21) as the metric of the largest manifold which metrics given only either in terms of $V$ or in terms of $W$ can be isometrically embedded. There will be then a maximal manifold on which metric (2.21) is $C^{2}$. One can also use the conformal treatment of the infinities to produce the Penrose diagram corresponding to the generalized de Sitter space we are dealing with. It is depicted in Fig. 1, where the regions 
labeled I describe the interval $0<r<r_{-}$, the regions labeled II describe the interval $r_{-}<r<r_{+}$, and the regions labeled III describe the spacetime within the exterior interval $r_{+}$ $<r<\infty$. This diagram is formed up as an infinite chain of asymptotically flat $(r \rightarrow 0)$ regions I which are connected to one another by means of regions II and III, and each region III is bounded by a timelike asymptotically flat infinite singularity at $r \rightarrow \infty$.

So far, we have confined ourselves to the case of a generalized de Sitter space with $\widetilde{Q}<0$ for a simply connected topology. One can also consider this spacetime with multiply connected topology on a given restricted region of it. This can be accomplished by imposing the symmetry of the Misner space [5] to the visualizing six-dimensional hyperboloid that embeds the generalized de Sitter space, that is by letting the Minkowski coordinates of that hyperboloid satisfy the identification property

$$
\begin{aligned}
& \left(z_{0}, z_{1}, z_{2}, z_{3}, z_{4}, z_{5}\right) \\
& \quad \leftrightarrow\left[z_{0} \cosh (n b)+z_{1} \sinh (n b), z_{0} \sinh (n b)\right. \\
& \left.\quad+z_{1} \cosh (n b), z_{2}, z_{3}, z_{4}, z_{5}\right],
\end{aligned}
$$

where $b$ is a dimensionless arbitrary period and $n$ is any integer number, $n=0,1,2, \ldots, \infty$. The boost transformation in the $\left(z_{0}, z_{1}\right)$ plane implied by identifications (2.23) will induce a boost transformation in the generalized de Sitter space. Hence, since the boost group in the generalized de Sitter space must be a subgroup of the generalized de Sitter group, the static metric (2.9) should also be invariant under the symmetry (2.23). One can readily see that this symmetry is in fact satisfied in the generalized de Sitter region covered by metric (2.9) and defined by $z_{1}>\left|z_{0}\right|$, where there are CTC's, with the boundaries at $z_{1}= \pm z_{0}$ and a given value of $\sum_{i=2}^{5} z_{i}^{2}$ being the Cauchy horizons that limit the onset of the nonchronal region from the causal exterior; that is the chronology horizons for the multiply connected generalized de Sitter space [7]. As applied to this space, the identifications (2.23) become

$$
t \leftrightarrow t+n b \kappa_{c}=t+\frac{n b\left(H^{2}+\sqrt{H^{4}-4 Q^{4}}\right)}{2 Q^{2} \sqrt{H^{2}-2 Q^{2}}},
$$

which applies only to the region defined by $r \leqslant r_{-}$. In the case of an extreme generalized de Sitter space, where $Q^{2}$ $=H^{2} / 2$, identifications (2.24) reduce to $t \leftrightarrow t+\infty$, a result which corresponds to the fact that the temperature of the extreme generalized de Sitter space is zero.

\section{THERMAL RADIATION IN GENERALIZED de SITTER SPACE}

In order to study the thermal properties of the generalized de Sitter space with $\widetilde{Q}<0$, which are to be expected because of the existence of event horizons in this space, we shall follow two distinct procedures. We shall first use the conventional Euclidean method [14], and then a new method based on calculating the Fourier transform of the Whitman function that corresponds to the propagation of a massless scalar field in the six-dimensional Minkowskian embedding space $[10,15]$.

We start with the Kruskal extension of the static metric which does not contain the geodesic incompleteness at the event horizons. In order for this metric to be definite positive we have to analytically continue the time coordinate according to the rotation $t=i \tau$. To see how this can be accomplished, we introduce new coordinates in the metric (2.21), such that

$$
\tan V^{\prime}=R+S, \quad \tan W^{\prime}=R-S .
$$

Then, the metric (2.21) becomes

$$
d s^{2}=-\frac{\left(1-H^{2} r^{2}+Q^{4} r^{4}\right)\left(d R^{2}-d S^{2}\right)}{\kappa_{c}^{2}\left(R^{2}-S^{2}\right)}+r^{2} d \Omega_{2}^{2},
$$

where $r$ is defined by

$$
S^{2}-R^{2}=\left[\left(\frac{r_{+}-r}{r_{+}+r}\right)^{r_{-} / r_{+}}\left(\frac{r_{-}+r}{r_{-}-r}\right)\right]^{r_{-} /\left(r_{+}+r_{-}\right)},
$$

so that the surface $r=0$ is defined by $S^{2}-R^{2}=1$, and the time $t$ results from

$$
\frac{R-S}{R+S}=-\exp \left(-2 \kappa_{c} t\right)
$$

In the region $r \leqslant r_{-}$, metric (3.2) becomes definite positive, provided we perform the continuation

$$
S=i \xi, \quad t=i \tau .
$$

Then, from Eq. (3.4) we obtain

$$
\xi+i R=-\sqrt{R^{2}+\xi^{2}} \exp \left(-i \kappa_{c} \tau\right) .
$$

We thus see that the Euclidean time $\tau$ becomes a periodic variable, with a period $2 \pi / \kappa_{c}$. Following then the usual procedure we can deduce that the generalized de Sitter space with $\widetilde{Q}<0$ is characterized by a background temperature given by

$$
T=\frac{\kappa_{c}}{2 \pi}=\frac{Q^{2} \sqrt{H^{2}-2 Q^{2}}}{\pi\left(H^{2}+\sqrt{H^{4}-4 Q^{4}}\right)},
$$

where it can be noted that in the extreme case $Q^{2}$ $=H^{2} / 2, T=0$, so that no thermal bath remains.

Although it reproduces the correct results, the Euclidean method is rather mysterious and much too schematic. To see the physics behind the thermal radiation process, we shall now follow a different procedure which relies on performing a calculation in the six-dimensional Minkowski space $[10,15]$, which is thereby taken to play the role of the maximum extension of the static metric, instead of the Kruskal space on which our previous discussion was made. 
As related to the embedding of the generalized de Sitter space with $\widetilde{Q}<0$, the Rindler wedge restriction of the sixdimensional Minkowski space requires the usual coordinate redefinitions

$$
z_{0}=\zeta \sinh (g \eta), \quad z_{1}=\zeta \cosh (g \eta),
$$

with all other $z_{m}$ remaining unchanged. Then,

$$
d s^{2}=-g^{2} \zeta^{2} d \eta^{2}+d \zeta^{2}+\sum_{m=2}^{5} d z_{m}^{2}
$$

The lines $\zeta=$ const, $z_{m}=$ const are the trajectories of the uniformly accelerated observers with constant acceleration $g$; i.e., those observers moving along the hyperbolas $\zeta^{2}=z_{1}^{2}$ $-z_{0}^{2}$. They will possess an event horizon located at $\zeta=0$. The coordinates $\eta, \zeta$ cover only the quadrant $z_{1}>z_{0}$, such as the coordinates $t, r$ do. One can pass from metric (3.8) to the static metric by using

$$
\eta=t, \quad \zeta=\frac{\sqrt{1-H^{2} r^{2}+Q^{4} r^{4}}}{\kappa_{c}},
$$

So as the relations for $z_{2}, z_{3}, z_{4}$, and $z_{5}$ given in Sec. II, and the identification $g=\kappa_{c}$. Because $\zeta=$ const represents a trajectory with constant proper acceleration, we conclude that a static observer ( $r, \theta, \phi$ constants) in the interior generalized de Sitter static geometry is a particular type of uniformly accelerated observer (with acceleration proportional to $\kappa_{c}$ ) in the embedding Minkowski space.

The power spectrum detected by an accelerated de Witt detector which probes the quantum fluctuations of a scalar massless field is given by [16]

$$
F(\omega) \int_{-\infty}^{+\infty} D\left(\eta, \eta^{\prime}\right) \exp (i \omega \triangle \eta) d(\triangle \eta),
$$

where $D\left(\eta, \eta^{\prime}\right)$ is the Whitman function $D\left(z, z^{\prime}\right)$ [14] evaluated along the world line of the detector, $\triangle \eta=\eta$ $-\eta^{\prime}$. There is now the problem that, although the world line of the detector is the same in Minkowski and generalized de Sitter spaces, the scalar field is not restricted to the twodimensional space, but extends over the full spacetime. In order to keep studying the behavior of the field close to the detector world line, we will make our calculation in the geometric optics approximation where only short wavelengths are taken into account [10]. Thus, in this approximation, the Whitman function for the world line (2.10) becomes

$$
\begin{aligned}
& D\left(\eta, \eta^{\prime}\right) \\
& \quad=-\frac{\kappa_{c}^{2}}{16 \pi^{2}\left(1-H^{2} r^{2}+Q^{4} r^{4}\right) \sinh ^{2}\left[\kappa_{c}(\triangle \eta-i \epsilon) / 2\right]} .
\end{aligned}
$$

Choosing as the integration contour in the $\Delta \eta$ complex plane the strip $0 \leqslant \operatorname{Im} \kappa_{c} \triangle \eta \leqslant 2 \pi$ and using the theorem of residues, we can evaluate the Fourier integral (3.10) to give

$$
F(\omega) \propto \frac{1}{\left(1-H^{2} r^{2}+Q^{4} r^{4}\right)\left[\exp \left(2 \pi \omega / \kappa_{c}\right)-1\right]},
$$

the proportionality factor being given by a certain product of powers of the frequency $\omega$ and the trajectory of the accelerated observer $\zeta$. In this approximation, the power spectrum is thus Planckian with a temperature given by expression (3.7). Making the same kind of calculation in the full sixdimensional Minkowski space, without resorting to geometric optics approximation, we finally obtain for the power spectrum

$$
F(\omega) \propto \frac{1+\kappa_{c}^{2} / \omega^{2}}{\exp \left(2 \pi \omega / \kappa_{c}\right)-1},
$$

which is not Planckian and only becomes so in the geometric optics approximation where $\omega \gg \kappa_{c}$. The proportionality factor in Eq. (3.13) also depends on a product of given powers of frequency $\omega$ and observer's trajectory $\zeta$. It is worth noting the dependence of $F(\omega)$ on $\zeta$ which reflects the feature that the horizons in our spacetime are observer-dependent, such as it happens in de Sitter space.

\section{GENERALIZED SCHWARZSCHILD-de SITTER SPACE}

In this section we briefly discuss the different possible first-order perturbations of the Ginsparg-Perry type [8] that can occur in both the singly degenerate and doubly degenerate cases corresponding to the generalized Schwarzschild-de Sitter space. Let us recall that the metric of this space can be defined by

$$
e^{A}=V(r)=1-H^{2} r^{2}+Q^{4} r^{4}-\frac{2 m}{r}
$$

In order to determine the positions of the horizons in the extreme (degenerate) case, we first obtain the extremals of the function $r V(r)$, i.e., we solve for $d[r V(r)] / d r=0$, which gives for $Q^{2}=H^{2} / 2$

$$
\left(r_{ \pm}^{0}\right)^{2}=\frac{2(3 \pm 2)}{5 H^{2}}
$$

The horizon at $r_{+}^{0}$ corresponds to the singly degenerate case (the two cosmological horizons coincide). From the horizon condition $V\left(r_{+}^{0}\right)=0$ we obtain then $m=0$, i.e., there is no black hole in this case, and $r_{+}^{0}$ exactly is the degenerate radius of the two cosmological horizons of the generalized de Sitter space studied in the precedent sections. The horizon at $r_{-}^{0}$ can be associated with the doubly degenerate case (the degenerate cosmological horizon also coincides with the radius of the black hole). Replacing $r_{-}^{0}$ into $V(r)=0$, we get then

$$
m=m_{0}=\frac{8}{5} r_{-}^{0}
$$


We consider now the distinct first-order perturbations of the type first considered by Ginsparg and Perry [8] for the above two degenerate cases. Let us first study perturbations of the doubly degenerate case where we first introduce the ansatz

$$
\begin{gathered}
t=\frac{\psi}{2 r_{-}^{0} H^{2} \delta}, \\
r=r_{-}^{0}\left(1-\delta \cos \chi+\frac{1}{13} \delta^{2}\right), \\
Q^{4}=H^{4}\left(\frac{1}{4}-\frac{40}{13} \delta^{2}\right),
\end{gathered}
$$

with $m_{0}$ unperturbed, and $0<\delta \ll 1$. In this case, we have

$$
V(\chi)=\frac{4}{5} \delta^{2} \sin ^{2} \chi
$$

so that at first order in $\delta$,

$$
d s^{2}=\frac{1}{2 H^{2}}\left(d \chi^{2}-\sin ^{2} \chi d \psi^{2}\right)+\left(r_{-}^{0}\right)^{2}(1+2 \delta \cos \chi) d \Omega_{2}^{2} .
$$

As fixed by $V(\chi)=0$, the horizons will appear at $\chi=0$ and $\chi=\pi$, which correspond to the perturbed cosmological horizons at $r_{+}=r_{-}^{0}(1+\delta)$ and $r_{-}=r_{-}^{0}(1-\delta)$, respectively. The radius of the black hole keeps being located at $r_{b}=r_{-}^{0}$ and it no longer is associated to an event horizon.

In order to evaluate the thermal properties of the soperturbed spacetime, we calculate the surface gravity $\kappa$ in each case, determining then the associated temperature by means of the simple expression $T=\kappa / 2 \pi$. In order to compute $\kappa$, we shall follow Bousso and Hawking [17], i.e., we set

$$
\kappa=2 \pi \frac{\gamma_{\psi}}{\psi_{h}^{i d}}
$$

where

$$
\gamma_{\psi}=H^{2} r_{-}^{0}
$$

and

$$
\psi_{h}^{i d}=\left.2 \pi \sqrt{g_{\chi \chi}}\right|_{\chi=\chi_{h}}\left(\frac{\partial}{\partial \chi} \sqrt{g_{\psi \psi}} \mid \chi=\chi_{h}\right)^{-1} .
$$

We then obtain

$$
T_{+}=\frac{H^{2} r_{-}^{0}}{2 \pi}, \quad T_{-}=-\frac{H^{2} r_{-}^{0}}{2 \pi}, \quad T_{b}=0 ;
$$

i.e., the black hole with intermediate size would not radiate, but there is a radiation process transfering energy from the outer to the inner horizon of the generalized de Sitter space, until the double degeneracy is restored. Thus, the system appears to be stable to these perturbations.

We next consider perturbations of the form

$$
\begin{gathered}
r=r_{-}^{0}\left(1-\delta \cos \chi-\delta^{2}\right), \\
m=m_{0}\left(1+\frac{11 H r_{-}^{0} \delta^{2}}{8}\right), \\
Q^{4}=H^{4}\left(\frac{1}{4}-2 \delta^{2}\right),
\end{gathered}
$$

with the time coordinate $\psi$ as defined by the first of Eqs. (4.4). Hence, one again obtains Eqs. (4.5) and (4.6) for $V(\chi)$ and the perturbed metric, respectively. Thus, the horizons at $\chi=0, \pi$ will correspond to the respective radii $r_{h}(\chi=0)$ $=r_{-}^{0}(1+\delta)$ and $r_{h}(\chi=\pi)=r_{-}^{0}(1-\delta)$. This may describe two distinct situations. Either (a) $r_{h}(\chi=0)=r_{b}$ (radius of an evaporating black hole) and $r_{h}(\chi=\pi)=r_{-b}$ (radius of an antievaporating black hole [18]), with the degeneracy of the cosmological horizons preserved at $r_{ \pm}=r_{-}^{0}$, or (b) $r_{h}(\chi)$ $=r_{+}=r_{b}$ and $r_{h}(\chi=\pi)=r_{-}=r_{-b}$, i.e., the degeneracy of the cosmological horizons is broken, but that between the horizons of the evaporating (antievaporating) black hole and the cosmological outer (inner) horizons is preserved. In case (a) the temperatures of the black holes, as calculated by using Eqs. (4.7)-(4.9), are $T_{ \pm b}= \pm H^{2} r_{-}^{0} / 2 \pi$, and those of the degenerate cosmological horizons are $T_{ \pm}=0$. In case (b), we obtain instead, $T_{ \pm b}=T_{ \pm}= \pm H^{2} r_{-}^{0} / 2 \pi$. It follows that in any of the two cases, our spacetime is stable to the perturbations specified by Eqs. (4.10).

Another kind of possible perturbations in the doubly degenerate case can finally be considered. It would describe perturbations which always leave the degeneracy between the cosmological horizons unchanged, and is defined by the same time perturbation as in Eqs. (4.4) and (4.10), and

$$
\begin{aligned}
& r=r_{-}^{0}(1+\delta \cos \chi), \\
& m=m_{0}\left(1-\frac{5}{4} \delta^{2}\right),
\end{aligned}
$$

$$
Q^{4}=\frac{1}{4} H^{4}
$$

These perturbations again lead to Eqs. (4.5) and (4.6) for $V(\chi)$ and the perturbed metric. In this case, the temperatures that result are $T_{ \pm}=0$ and $T_{ \pm b}= \pm H^{2} r_{-}^{0} / 2 \pi$, so that the spacetime is once again stable to the perturbations.

Let us consider in what follows of this section the case in which the horizon is at $r=r_{+}^{0}$, with $m=0$. It describes a singly degenerate generalized de Sitter space with negative $\widetilde{Q}$ and no black hole being initially present. We introduce then the perturbative parameter $\delta$ by means of the equation

$$
Q^{4}=H^{4}\left(\frac{1}{4}-\frac{2}{5} \delta^{2}\right), \quad 0 \leqslant \delta \ll 1
$$


The singly degenerate case corresponds to the limit $\delta \rightarrow 0$. The new time and radial coordinates $\psi$ and $\chi$, will now be given by

$$
t=\frac{\psi}{2 r_{+}^{0} H^{2} \delta}, \quad r=r_{+}^{0}(1+\delta \cos \chi) .
$$

We then let the perturbation induce creation (or annihilation) of an extremely tiny black hole with mass

$$
m=\frac{6 r_{+}^{0} \delta^{2}}{5} .
$$

The inner and outer horizons of the generalized de Sitter space lie, respectively, at $\chi=\pi$ and $\chi=0$, while the black hole horizon will be at $2 \mathrm{~m}$. To first order in $\delta$, from the above transformations, we obtain the new metric

$$
\begin{aligned}
d s^{2}= & \frac{1}{2 H^{2}}\left[(1+2 \delta \cos \chi) \sin ^{2} \chi d \psi^{2}\right. \\
& \left.-(1-2 \delta \cos \chi) d \chi^{2}\right]+\frac{2}{H^{2}}(1+2 \delta \cos \chi) d \Omega_{2}^{2} .
\end{aligned}
$$

We notice that, since $V(r)=1-H^{2} r^{2}+Q^{4} r^{4}$ becomes negative when it is perturbed, i.e.,

$$
V(\chi)=-4 \delta^{2}(1+2 \delta \cos \chi) \sin ^{2} \chi,
$$

the coordinate $\psi$ becomes spacelike and the radial coordinate becomes timelike. In these coordinates, the topology of the sections at constant $\psi$ is $S^{1} \times S^{2}$, and this means creation of a handle. For these solutions, the radius of the two spheres $r$ varies along the $S^{1}$ coordinate $\chi$, with the minimal (maximal) two sphere corresponding to the inner (outer) cosmological horizon, and the ergoregion between the cosmological horizons nesting a little black hole of radius $2 m$ much smaller than the size of that ergoregion $r_{+}-r_{-}=2 r_{+}^{0} \delta$. At the degenerate case, the metric (4.14) becomes

$$
d s^{2}=\frac{1}{2 H^{2}}\left(\sin ^{2} \chi d \psi^{2}-d \chi^{2}\right)+\frac{2}{H^{2}} d \Omega_{2}^{2} .
$$

This metric is not the same as the Nairai solution. One still can recover a metric with the conventional signature by rotating $\chi=i \sigma$, so that the line element (4.15) becomes

$$
\begin{aligned}
d s^{2}= & -\frac{1}{2 H^{2}}\left[(1+2 \delta \cosh \sigma) \sinh ^{2} \sigma d \psi^{2}\right. \\
& \left.-(1-2 \delta \cosh \sigma) d \sigma^{2}\right]+\frac{2}{H^{2}} \\
& \times(1+2 \delta \cosh \sigma) d \Omega_{2}^{2},
\end{aligned}
$$

and for the degenerate case

$$
d s^{2}=-\frac{1}{2 H^{2}}\left(\sinh ^{2} \sigma d \psi^{2}-d \sigma^{2}\right)+\frac{2}{H^{2}} d \Omega_{2}^{2} .
$$

Metric (4.18) would represent a wormhole with the throat at the outer horizon. If we Wick rotate the time variable, that is $\psi=i \varphi$, we finally obtain a metric with Euclidean signature. In the degenerate case, this will produce two round two spheres of different radius.

The interpretation of these perturbations becomes again twofold. One can regard them as representing a breaking of the degeneracy between the cosmological horizons at $r_{ \pm}$, in which case

$$
\begin{aligned}
& r_{h}(\chi=0)=r_{+}^{0}(1+\delta)=r_{+}=r_{b}, \\
& r_{h}(\chi=\pi)=r_{+}^{0}(1-\delta)=r_{-}=r_{-b} .
\end{aligned}
$$

On the other hand, we can also look at these perturbations as preserving that degeneracy, so that $r_{h}(\chi=0)=r_{b}, r_{h}(\chi$ $=\pi)=r_{-b}$, and $r_{ \pm}=r_{+}^{0}$ (as evaluated at $\chi=\pi / 2$ ).

The calculation of the surface gravity for these perturbations follows the same line as for the doubly degenerate cases, unless for the parameter $\gamma_{\psi}$ which should now be given by

$$
\gamma_{\psi}=H^{2} r_{+}^{0},
$$

instead of Eq. (4.8). We then obtain for the temperatures corresponding to the two horizons $r_{h}$

$$
\begin{gathered}
T(\chi=0)=\frac{H^{2} r_{+}^{0}(1+2 \delta)}{2 \pi}, \\
T(\chi=\pi)=-\frac{H^{2} r_{+}^{0}(1-2 \delta)}{2 \pi},
\end{gathered}
$$

and for $\chi=\pi / 2$,

$$
T\left(\chi=\frac{\pi}{2}\right)=-\frac{H^{2} r_{+}^{0} \delta}{2 \pi} .
$$

When the degeneracy between the cosmological horizons is broken by the perturbations, then $T_{b}=T_{+}=T(\chi=0), T_{-b}$ $=T_{-}=T(\chi=\pi)$, while the temperature (4.22) measures a thermal process by which the vacuum itself antiradiates. In the case for which the above degeneracy is preserved, we obtain instead, $T_{b}=T(\chi=0), T_{-b}=T(\chi=\pi)$, and $T_{ \pm}$ $=T(\chi=\pi / 2)$. All of these thermal processes lead to a final state in which there is no black hole and the generalized de Sitter space becomes finally again degenerate, so also these perturbations are stable.

\section{SUMMARY AND CONCLUSIONS}

In this paper we argue that the most general solution to the general-relativity field equations that describe an empty spherically symmetric spacetime must contain two constant parameters in order to account for the quantum vacuum effects. According to the possible combinations of the signs of 
such parameters, the general solution splits into three essentially different static spacetimes, two describing generalized de Sitter spaces and one describing a generalized anti-de Sitter space. In this paper, we have confined ourselves to the study of the generalized de Sitter case (positive cosmological constant) with a negative additional constant whose physical dimensions are the same as those of a space curvature squared. Such a solution possesses two event horizons and can be visualized as a six hyperboloid with embedding Minkowskian metric. It is maximally extensible by defining Kruskal-like coordinates, and corresponds to a spacetime which can be made multiply connected inside the inner event horizon by imposing the symmetry of the Misner space.

Since the additional vacuum constant term in the righthand side of the Einstein equations ought to correspond to an existing curvature squared terms in the left-hand side of such equations, it could be thought that, as corresponding to all other perturbation terms in the gravitational Lagrangian of a Lovelock theory [19], one could have an arbitrary number of additional constants characterizing quantum fluctuations of vacuum. From this point of view, the solution studied in this paper might be regarded as merely being nothing but the second-order semiclassical approximation from the most general full solution.

The thermal properties of the spacetime dealt with in this work have been studied by using both the conventional Euclidean procedure, and a novel method based on considering the propagation of a massless scaler field in its sixdimensional embedding space, where the thermal spectrum is computed as the Fourier transform of the Whitman equation. We obtain that an observer moving along the origin of the radial coordinates will detect a background thermal bath at a temperature which vanishes only as one approaches the extreme (degenerate) case.

We have finally studied the stability of the generalized
Schwarzschild-de Sitter spacetime to the allowed GinpargPerry perturbations in first-order approximation. It has been obtained that this spacetime perdures to all types of the considered perturbations, and that in the case that no balck hole was initially present, processes are allowed in which black hole pairs are created and then evaporated away.

There are two ways along which the present work could be continued. On the one hand, it appears appropriate to investigate the cosmological effects that the additional vacuum term may have in cosmological models. One should then consider the global Friedman-Robertson-Walker metric that can be associated with the static metric studied in this work. On the other hand, one would also investigate the effects that can be expected from inserting a positive vacuum additional term in the properties of the anti-de Sitter space. We have already obtained that in this case, the generalized anti-de Sitter space has an event horizon and, therefore, one should expect that, contrary to what happens in the usual anti-de Sitter space, this space has a precise temperature, and that its properties are similar to those of the generalized de Sitter space with positive $\widetilde{\mathrm{Q}}$.

Note added in proofs. Equation (2.7) is really a solution to all field equations provided we add a factor 2 in front of the extra term $\widetilde{Q} r^{2}$ in the components $(r r)$ and $(t t)$ of Eqs. (2.6). Such a factor is in these cases actually required to account for the breakdown of homgeneity of the spatial sections induced by the $r^{2}$ dependence of the extra term and the invariance of the metric under reflection of $r$ and $t$, and has been in fact obtained by computer calculation kindly performed by Professor A. G. Agnese in Genova University.

\section{ACKNOWLEDGMENTS}

This research was supported by DGICYT under Research Project No. PB97-1218.
[1] W. de Sitter, Proc. K. Ned. Akad. Wet. 19, 1217 (1917); 20, 229 (1917).

[2] C. Kahn and F. Kahn, Nature (London) 257, 451 (1975).

[3] A. Einstein, Preuss. Akad. Wiss. Berlin Sitzber. 142 (1917) [English translation in H. A. Lorentz, A. Einstein, H. Minkowski, and H. Weyl, The Principles of Relativity. A Collection of Original Memoirs on the Special and General Theory of Relativity (Dover, New York, USA, 1952)].

[4] W. Rindler, Essential Relativity (Springer-Verlag, New York, 1977).

[5] S. W. Hawking and G. F. R. Ellis, The Large Scale Structure of Space-Time (Cambridge University Press, Cambridge, England, 1973).

[6] E. S. Schrödinger, Expanding Universe (Cambridge University Press, Cambridge, England, 1956).

[7] J. R. Gott and Li-Xin-Li, Phys. Rev. D 58, 023501 (1998); see also P. F. González-Díaz, ibid. 59, 123513 (1999).

[8] P. Ginsparg and M. J. Perry, Nucl. Phys. B222, 245 (1983).

[9] H. Stephani, in Unified Field Theories in More Than Four
Dimensions, edited by V. de Sabbata and E. Schmutzer (World Scientific, Singapore, 1983).

[10] M. Beciu and H. Culetu, Mod. Phys. Lett. A 14, 1 (1999).

[11] Ya. B. Zel'dovich, Zh. Éksp. Teor. Fiz. Pis'ma 6, 883 (1967) [JETP Lett. 6, 316 (1967)].

[12] B. S. DeWitt, in General Relativity. An Einstein Centenary Survey, edited by S. W. Hawking and W. Israel (Cambridge University Press, Cambridge, England, 1979).

[13] M. D. Kruskal, Phys. Rev. 119, 1743 (1960).

[14] N. D. Birrell and P. C. W. Davies, Quantum Fields in Curved Spaces (Cambridge University Press, Cambridge, England, 1982).

[15] M. Bouhmadi and P. F. González-Díaz (unpublished).

[16] D. W. Sciama, P. Candelas, and D. Deutsch, Adv. Phys. 30, 327 (1981).

[17] R. Bousso and S. W. Hawking, Phys. Rev. D 54, 6312 (1996).

[18] R. Bousso and S. W. Hawking, Phys. Rev. D 57, 2436 (1998).

[19] D. Lovelock, J. Math. Phys. 12, 498 (1971). 